\title{
Ricciarda Ricorda
}

\section{Ana Cecilia Prenz Kopušar, «un’argentina italiana nata a Belgrado»}

Riassunto: Ana Cecilia Prenz (1964), nel volume del 2016, Attraversando il fiume in bicicletta, si misura con tre identità, argentina, serba e italiana, e con le rispettive culture e lingue, in una storia personale che attraversa momenti cruciali del Novecento, come la dittatura argentina, il periodo di Tito, le tensioni politiche della storia italiana degli anni Settanta. Il diario, scritto prima in spagnolo e poi tradotto dall'autrice stessa in italiano, presenta pertanto un'inedita commistione di luoghi, situazioni, condizioni, con il racconto delle vicende e della formazione straordinaria di una giovane che ha dovuto di volta in volta ricominciare la propria vita, accettando di riconoscersi un'identità dotata di più connotazioni. L'analisi del testo punterà sul tema del confronto e dell'incontro tra le diverse dimensioni, con la ricostruzione di un'identità che deve ridefinirsi dopo strappi e vicende laceranti.

\section{Ana Cecilia Prenz Kopušar, «an Argentinean from Italy born in Belgrade»}

\begin{abstract}
Ana Cecilia Prenz (1964), in the volume of 2016, Attraversando il fiume in bicicletta, is measured by three identities, Argentinean, Serbian and Italian, and their respective cultures and languages, in a personal history that crosses crucial moments of the twentieth century, such as the Argentine dictatorship, the period of Tito, the political tensions of Italian history in the seventies. The diary, written first in Spanish and then translated into Italian by the author herself, therefore presents an unusual mixture of places, situations, conditions, with the story of the events and the extraordinary formation of a young woman who had to start her life over again, accepting to recognize her identity with more connotations. The analysis of the text will focus on the theme of comparison and encounter between the different dimensions, with the reconstruction of an identity that must redefine itself after tears and lacerating events.
\end{abstract}

2 Open Access. (C) 2020 Ricciarda Ricorda, published by De Gruyter. ((c))BY-NC-ND This work is licensed under the Creative Commons Attribution-NonCommercial-NoDerivatives 4.0 License.

https://doi.org/10.1515/9783110640069-016 
«In tutto quello che faccio ho sempre cercato di unire i miei tre mondi, Jugoslavia, Argentina e Italia. Li vorrei vivere contemporaneamente. Di recente ho detto a mio figlio: "esiste la parola terbiqua?” Ecco, io sono così». ${ }^{1}$

Nel caso di Ana Cecilia Prenz Kopušar la coesistenza di nazionalità diverse non si realizza in un luogo, in una dimensione fisica, ma all'interno della sua stessa persona che, come ha precisato lei medesima, con un neologismo che bene sintetizza la sua condizione, «terbiqua», appartiene a tre dimensioni, che vorrebbe vivere contemporaneamente, perché, se lo spostamento è sofferenza, è il dolore di lasciare una parte di sé altrove, l'unica possibilità di non continuare a percepire tale parte come mancanza, come nostalgia, è di portarla dentro di sé e continuare così a farla vivere.

Tale condizione «terbiqua» è al centro del suo libro Attraversando il fiume in bicicletta, pubblicato prima in Argentina, dove si sono succedute due edizioni, nel 2013 e nel 2015, con il titolo Cruzando el río en bicicleta, e quindi, nel 2016, tradotto da lei stessa in italiano, con alcuni cambiamenti: sono diverse infatti le pagine finali, che presentano alcune omissioni e aggiungono una conclusione di tipo riflessivo, e sono introdotte alcune immagini, fotografie di oggetti dotati di una valenza simbolica in relazione al contesto e dell'autrice da piccola con il padre. $^{2}$

Il testo racconta le vicende e le esperienze di Ana Cecilia, che l'hanno vista muoversi tra tre diverse appartenenze: è proprio il senso dell'appartenenza ad accamparsi centralmente nell'opera, già nel titolo del primo capitolo, appunto Appartenenza. Sembra significativo che la scrittrice preferisca in genere questa parola a identità, «parola avvelenata», per usare l'attributo conferitole da Francesco Remotti, "perché promette ciò che non c'è; perché ci illude su ciò che non siamo; perché fa passare per reale ciò che invece è una finzione o, al massimo, un'aspirazione». ${ }^{3}$

1 Arianna Boria, Ana Cecilia Prenz, «I miei tre mondi dentro un diario», «Il Piccolo», 21 aprile 2015.

2 Queste le edizioni: Ana Cecilia Prenz Kopušar, Cruzando el río en bicicleta, Mediterránea, Centro di Studi Interculturali, Dipartimento di Studi Umanistici, Università di Trieste, 2013; Buenos Aires, De la Tablita Dorada, City Bell, 2015; Attraversando il fiume in bicicletta, Trieste, Vita Activa, 2016. Sulle differenze tra l'edizione in spagnolo e quella in italiano cfr. Adriana Cristina Crolla, Migración, autoficción y autotraducción en Cruzando el río en bicicleta de Ana Cecilia Prenz Kopušar, «Oltreoceano», 13, 2017, pp. 197-206, e Susanna Regazzoni, Entre Argentina e Italia pasando por Yugoslavia: Cruzando el río en bicicleta de Ana Cecilia Prenz Kopušar, «Ars \& Humanitas», 2017, vol. 11, pp. 220-231.

3 Francesco Remotti, L'ossessione identitaria, Bari, Laterza, 2017 (2010), p. 7. 
Del resto, Octavio Prenz, padre di Ana Cecilia, «curioso esempio di scrittore sudamericano-mitteleuropeo», come lo ha definito Claudio Magris, è autore del romanzo Solo gli alberi hanno radici (2013), ambientato a Ensenada de Barragán, paese argentino del quale scrive anche Ana Cecilia e in cui, per usare ancora le parole di Magris, «arrivano, partono, ritornano emigranti di diverse generazioni, provenienti soprattutto dall'Istria italo-croata. Ma non si tratta di sradicati, perché - come Prenz ha detto più volte anche di se stesso - non si sentono alberi strappati alla loro terra, ma piuttosto animali randagi che amano vagabondare per il mondo e amano ogni luogo in cui sostano, per breve tempo o a lungo o per sempre, aperti a nuovi incontri, mescolanze, congedi». ${ }^{4}$ Come già il titolo suggerisce, il romanzo propone dunque una visione del mondo alternativa alla fissazione identitaria denunciata da Remotti, vicina invece a quella sottostante alle pagine della figlia.

Qualche dato sulla scrittrice: docente presso l'Università degli Studi di Trieste, si occupa di letteratura teatrale in lingua spagnola, in particolare della drammaturgia e del teatro argentino contemporaneo, di autori teatrali spagnoli del Rinascimento e della letteratura teatrale 'judeoespañola' nei Balcani. Il suo interesse per il tema dell'interculturalità è costante e si articola in diverse attività: nel campo del teatro e della letteratura è attestato dalla pubblicazione di articoli e saggi sulla scrittrice sefardita della Bosnia Laura Papo Bohoreta e dalle traduzioni di autori argentini, italiani, sloveni e bosniaci, tra cui il poeta argentino José María Pallaoro, il drammaturgo argentino Eduardo Pavlovski e l'attrice, poetessa e drammaturga slovena Saša Pavček. Cura inoltre in Slovenia il centro culturale La Casa de Kamna, luogo di incontro e riflessione sull'America Latina; coordina anche gli accordi di cooperazione scientifica, didattica e culturale dell'Università di Trieste con quelle di Sarajevo e del Paraguay.

4 Claudio Magris, Prefazione. Un mitteleuropeo sudamericano, in Juan Octavio Prenz, Solo gli alberi hanno radici, traduzione di Betina Lilián Prenz, Milano, La nave di Teseo, 2017, ebook posizione 9-21. Juan Octavio Prenz, nato in Argentina, vissuto in Jugoslavia, approdato infine a Trieste, con la famiglia, docente universitario, è scrittore, saggista, poeta e traduttore: tra i suoi romanzi, con quello citato, si ricordano La favola di Innocenzo Onesto, il decapitato (2001) e Il signor Krek (2014); tra i libri di poesia, La Santa Pinta de la Niña Maria (1992), con cui ha ottenuto il Premio Internazionale «Casa de las Américas». Betina Prenz, traduttrice di Solo gli alberi hanno radici e del Signor Krek, studiosa di filosofia del linguaggio, è sorella di Ana Cecilia e personaggio del suo testo. 
L’approdo di Ana Cecilia Prenz a Trieste risale al 1979: nata a Belgrado (Serbia) nel 1964, da padre argentino ma di famiglia croato-istriana e madre pure argentina, di ascendenza franco-spagnola, dopo pochi anni ritorna con i suoi a La Plata, in Argentina, dove trascorre l'infanzia e la prima adolescenza; nel 1975, quando la situazione inizia a farsi confusa e a manifestarsi l'attività dell'Alianza Anticomunista Argentina, con minacce di morte al padre, il cui nome viene inserito in una 'lista nigra', la famiglia lascia il paese e si stabilisce a Belgrado, dove Ana Cecilia frequenta la scuola media. Anche in Serbia, dopo qualche anno, i Prenz capiscono che «qualcosa non andava nel migliore dei modi» e decidono di spostarsi in Italia, scegliendo Trieste in quanto vicina alla Jugoslavia e, dichiara la scrittrice, perché ritenevano che «la mentalità italiana era vicina a quella argentina». ${ }^{5}$ Dopo il liceo, decide di proseguire gli studi a Roma, alla Sapienza, dove si laurea in Discipline dello Spettacolo, mentre consegue il dottorato di ricerca all'Università de La Plata in Argentina, ritrovando un altro modo, altre vie, il teatro, la letteratura, per entrare nella storia del paese, per un lento recupero di quella dimensione. ${ }^{6}$

Nella storia della famiglia Prenz si verifica dunque un incrocio di appartenenze e di destini, che Ana Cecilia racconta nel loro dispiegarsi: una sicura vena autobiografica - gli eventi raccontati appartengono tutti alla storia della sua famiglia, i personaggi messi in scena pure, dai nonni materni e paterni, ciascuno seguito nelle sue vicende e fatto oggetto di un ritratto tanto puntuale quanto suggestivo, soprattutto le due nonne, agli zii e ai parenti tutti, agli amici, nominati con precisione - lascia però anche spazio a una dimensione creativa, soprattutto nella particolare articolazione del discorso, che non segue la cronologia dei fatti, ma li disarticola, mescolando tempi ed episodi. Non vi è dubbio pertanto che si tratti di un testo di difficile collocazione, per una simile commistione di autobiografia e di finzione, ma anche per la volontà dell'autrice di raccontare, attraverso la propria vicenda esistenziale, una storia più ampia, di paesi e persone che hanno vissuto la guerra, la dittatura, la lotta per la democrazia. Mi pare però che il testo non si risolva del tutto nella dimensione dell'auto-finzione, almeno nell'accezione italiana, genere ibrido di cui attualmente molto si discute e a cui alcune studiose di area ibero-americana lo ascrivono, perché non mi sembra che l'autrice giochi con i piani dell'identità e

5 A. Boria, Ana Cecilia Prenz, «I miei tre mondi dentro un diario», cit.

6 «È stato lento il recupero del paese. I ritorni diluiti. Ogni volta cercavo di apprezzare qualcosa di nuovo. Lo studio mi ha permesso di entrare attraverso altri canali nella storia argentina e scoprire la terra di coloro che parlano poco, o forse, parlano molto, però li si ascolta poco» (A. C. Prenz Kopušar, Attraversando il fiume in bicicletta, cit., p. 96). 
non intenda rendere consapevolmente difficile per il lettore distinguere la realtà dalla finzione, il suo io reale da quello fittizio: si tratta semmai della tendenza ineludibile dell'autore dell'autobiografia a costruirsi come personaggio. ${ }^{7}$

Il racconto si allontana comunque dai tempi della fabula fin dall'inizio: l'avvio si colloca infatti negli anni dell'adolescenza a Belgrado, ma solo in un secondo tempo si apprenderà che si tratta, per la giovane Ana Cecilia, di un ritorno alla terra in cui è nata, anche se solo per un temporaneo spostamento della sua famiglia; nel capitolo subito successivo, invece, una sorta di flash back riporta il lettore alla precedente fase della vita della narratrice in Argentina, negli anni della scuola elementare, un istituto un po' hippie e molto antiautoritario. Il movimento pendolare da un tempo più recente a una dimensione anteriore continua, con un passaggio di nuovo a Belgrado, seguito da un ulteriore spezzone argentino, con il racconto della vita della nonna paterna, María, emigrata dal suo paese in Istria al Rio della Plata, e il riferimento alla famiglia materna. Anche l'approdo a Trieste lascia aperto il via-vai con la Jugoslavia, con una serie di ritorni a Belgrado che evocano anche nel presente una serie di immagini e ricordi del passato. Questo gioco complesso all'interno della dimensione cronologica è poi determinante ai fini della costruzione di una personalità, quella dell'io narrante, che si forma proprio nell'intreccio di diverse appartenenze, in una dialettica tra dimensioni differenti, tutte ugualmente importanti e necessarie.

Migrazione, esilio, appartenenza sono dunque al centro del racconto e vengono declinati secondo ottiche diverse: la lingua, i suoni, gli spazi, i documenti ufficiali. La lingua non è per caso al primo posto, perché la scrittrice la indica in più occasioni come fattore fondante del senso di appartenenza:

È stata bella la mia adolescenza in Jugoslavia. [...] Mai, come allora, ho percepito in modo così forte il senso di appartenenza. E pensare che ero piccola; non piccola, giovincella. Quanto basta per capire che ero una persona. Ho preso coscienza di me.

I miei compagni di classe mi insegnavano delle parole [...]. Ma quel Cecilia, che loro pronunciavano Zezilia e che scrivevano Cecilija, risultava complicato. Allora Dragana ha

7 Cfr. Philippe Lejeune, Il patto autobiografico, trad. it. Bologna, il Mulino, 1986. Ascrivono il testo all'ambito dell'autofinzione S. Regazzoni, Entre Argentina e Italia pasando por Yugoslavia: Cruzando el río en bicicleta de Ana Cecilia Prenz Kopušar, cit., pp. 221-223, e A. C. Crolla, Migración, autoficción y autotraducción en Cruzando el río en bicicleta de Ana Cecilia Prenz Kopušar, cit., pp. 198-199. La stessa Prenz, per altro, in un'intervista a Crolla, citata dalla medesima, ivi, p. 199, acconsentirebbe a tale collocazione: «Sí, creo que estamos ante un texto auto-ficcional». 
detto: previše teško, troppo difficile; od danas ti si Ceca, da oggi in poi tu sarai Zeza. E da allora per i miei amici a Belgrado sono diventata Ceca. Così mi hanno assimilato al loro mondo. Un atto semplice ma per me molto significativo. Ecco il senso di appartenenza. Dal primo giorno, anche se non parlavo la lingua, sono diventata una di loro. ${ }^{8}$

L'assimilazione al mondo degli altri, l'integrazione nella nuova dimensione passa dunque, prima di tutto, attraverso le parole, mentre fondamentale risulta l'atto magico della nominazione, l'invenzione del nome, nel linguaggio privato del gruppo di amici.

Numerosi sono poi nel testo i riferimenti alla lingua, alle diverse lingue che convivono in Ana Cecilia, senza confliggere, anzi, concorrendo a organizzare diversi tipi di immaginario e salvaguardando diverse esperienze esistenziali: così a Belgrado rimane vivo in lei lo spagnolo, che parla con piacere con l'amica della madre Joaquina e con la famiglia di Rubén, con cui lei e i suoi cercano anche in quel luogo diverso i loro «momenti argentini» - soprattutto i genitori, che «avevano bisogno di comunicare nella loro lingua»: ${ }^{9}$ eppure, proprio i genitori avevano sempre pensato che fosse necessario vivere secondo le abitudini del paese in cui si abitava, immergendo quindi le figlie nel mondo jugoslavo e facendo loro condividere tutto di quel mondo, «la lingua, gli spazi, le abitudini, le allegrie e i dolori» - ma qualche cosa di «nostro» rimaneva in loro, probabilmente proprio la lingua, quella materna, delle prime parole, lo spagnolo - lingua sua, di sua sorella, di sua madre. ${ }^{10}$

Nonostante questa sorta di 'prelazione' dello spagnolo, che è l'unica lingua in cui la narratrice dice di riuscire a parlare quando la stanchezza la coglie, anche le altre che appartengono alla sua vita le sono altrettanto indispensabili, le danno il senso del rapporto necessario fra il tutto e le parti:

La precarietà della nostra esistenza. Non l'ho mai percepita con angoscia, al contrario, con estrema bellezza.

E le lingue, ognuna a modo suo, con le proprie specificità, mi hanno condotto a tutto ciò, verso il tutto e verso il nulla, come un gioco.

Adoro parlare in serbo, la lingua dei forti, decisa, chiara, rotonda, senza inflessioni. Il nostro argentino, allegro, spensierato, diretto, creativo. L'italiano, elegante, sì, molto elegante, tanto elegante che diventa contorto, quanto contorto! ${ }^{11}$

8 A. C. Prenz Kopušar, Attraversando il fiume in bicicletta, cit., pp. 7-8.

9 Ivi, p. 36

10 Ivi, pp. 41-42.

11 Ivi, p. 43. 
L'incrocio delle diverse lingue, dunque, lungi dal creare difficoltà, è vissuto come elemento di ricchezza, spazio privilegiato; ed è una situazione che non riguarda solo Ana Cecilia, ma anche la sua famiglia; così il figlio Felipe, in un dialogo con il suo maestro di violino argentino:

- Tuo papà è sloveno e tua mamma di dove è?

- È argentina ma è nata a Belgrado.

- Ah, è serba?

- No, è argentina.

- No, è serba, dice il professore. Se tu sei nato in Italia, sei italiano. Io sono nato in Argentina, sono argentino, quindi, tua mamma, se è nata in Serbia, è serba.

- Felipe ammutolisce e pensa. Non capisce il ragionamento. Da quando è nato per lui sua madre è argentina.

- $\quad$ E che lingua parla tuo papà?

- Sloveno.

- E tua mamma?

- Spagnolo. Ma noi a casa parliamo molte lingue: io con mio fratello parlo sloveno, con mio papà italiano, con mia mamma spagnolo e mia mamma e mio papà tra loro parlano in serbo. E mio fratello con mia mamma parla in spagnolo e con mio papà sloveno. ${ }^{12}$

La lingua, per altro, è sì 'dono' materno, ma è anche lenta costruzione, a cui collabora altrettanto fortemente il contesto, la lingua che la narratrice definisce del «nostro intorno»; ed è un dramma se viene rifiutata, come le succede quando, durante la guerra, lavora come traduttrice e si trova a dover tradurre in italiano il discorso del ministro della neo Croazia: percepito il suo accento serbo, l'uomo rifiuta la sua traduzione e per lei, di sangue croato, anche se nata a Belgrado, è un primo schiaffo - dichiara - forte, molto forte; la lingua per la prima volta non è più un fattore di inclusione, ma diventa causa di divisione, di ostilità. ${ }^{13}$

Nel racconto, prevale però decisamente la prospettiva che vede nella lingua un elemento fondamentale di riconoscimento: lo conferma la storia della nonna paterna María, ripercorsa in due suggestivi capitoli; il primo, Buenos Aires 9, risale al tempo della migrazione, con il racconto del suo viaggio dal paese istriano-croato d'origine, Podmerišče, vicino a Pisino, all'Argentina, e con la riflessione, al Museo Hotel degli Immigrati a Buenos Aires, sull'esperienza di quella giovane partita da sola per una vita tutta da costruire. Un po' casuale

12 Ivi, p. 44.

13 Ivi, p. 109. 
risulta il suo approdo alla piccola città di Ensenada, dove si stabilisce e dove trova lavoro, nella fiorente - a quel tempo - industria per la lavorazione della carne; qui si fa una famiglia, cresce due figli maschi, che avrebbero entrambi studiato e sarebbero diventati socialisti, impegnati, continuando a lavorare fuori e in casa.

Anche per la nonna María la lingua è fattore di identificazione e di inclusione; con qualche effetto curioso: parla infatti in genere lo spagnolo, quello degli immigrati, ma, una volta che torna in Istria - con un viaggio premio offertole dall'associazione dei Giuliani nel mondo, in quanto una delle più anziane ensenadensi - insieme a una vicina di casa, pure istriana, con la quale ha sempre parlato spagnolo, passa a comunicare con lei solo e rigorosamente in dialetto istriano. Fin da subito, quando, giovane da poco immigrata, andava al lavoro a Berisso, al di là del fiume, un braccio del Rio della Plata, si era trovata immersa in una commistione singolare di lingue:

Colori intensi, alberi vicino al fiume e una moltitudine di gente che si dirigeva al lavoro. Le lingue, sempre le lingue, mormoravano nella memoria di mia nonna. Degli slavi: polacchi, russi, croati, sloveni; degli italiani, del nord e del sud. Tutte si compenetravano in quell’identità così argentina fatta dalle idiosincrasie d'ognuno. Ogni volta che María doveva scendere le scale per salire sull'imbarcazione, le dava fastidio che gli uomini le guardassero le mutande. Ci faceva ridere. Lei si arrabbiava. Quel breve tragitto creava in lei un'incomprensibile inquietudine interiore. Spesso sognava che attraversava il fiume in bicicletta galleggiando sull'acqua. ${ }^{14}$

L'ultima frase, per inciso, contiene il titolo del libro: il sogno raccontato dalla nonna, ha spiegato Ana Cecila Prenz, allude metaforicamente alla condizione in cui si riconosce lei stessa, è un'immagine del suo «andare e venire da uno spazio all'altro, anche con la testa»; e certo la narratrice compie continui spostamenti tra i diversi spazi del suo paesaggio dell'anima, a volte anche con qualche significativo corto-circuito.

Lo si può verificare a partire proprio dai luoghi, descritti sempre con forti connotazioni emotive: la Belgrado dell'adolescenza della narratrice «non era bella, era sporca, grigia, contaminata», però camminare per le sue strade per lei era bello, i suoi odori, il fumo, le sue fragranze, le sue case piene di vita, la musica, tutto l'attraeva, «come un magnete» e l'affascinava, conducendola a «tempi lontani, a una forma primigenia dell'umanità in cui la tenerezza, l'ingenuità, i buoni sentimenti e la brutalità si confondevano». ${ }^{15}$ La città continua a esercitare il suo fascino anche dopo il trasferimento a Trieste, e almeno fino alla

14 Ivi, p. 61.

15 Ivi, pp. 9-10. 
guerra, quando Ana Cecilia smette di visitare quella che era diventata ex Jugoslavia: fino ad allora invece ci ritorna tutte le volte che le è possibile, a riassaporarne gli aromi, a ritrovare il gusto dei diversi tipi di salsicce, a sentirsi parte di un gruppo, a condividere, a ossigenarsi; ${ }^{16}$ in un immaginario dialogo con una Jugoslavia personificata, la scrittrice immagina di sentirle dire «che tutte le cose finiscono a un certo punto», ma ribadisce la forza dei ricordi: «ho goduto della tua allegria. La tua voglia di condividere. Ti concedevi senza riguardi e mi dicevi che ti appartenevo. E io godevo dei tuoi odori, delle tue certezze». ${ }^{17}$

Più articolati i riferimenti ai paesaggi dell'Argentina, come bipartiti: da un lato, le immagini dell'infanzia, schermate, ma conservate intatte nella memoria, la casa dei nonni, luogo di pace, in cui svanivano tutti i conflitti, il verde intorno, il cortile; dall'altro, quelle scure, tetre, brutali della dittatura militare: un'Argentina piatta, in cui, tornandoci da ragazza, non si ritrova. ${ }^{18}$ Eppure, con il passare del tempo, riesce a riappropriarsi di questa nazionalità, torna a sentirsi anche argentina: le tre dimensioni della sua esistenza sono emblematicamente fissate nei suoi documenti ufficiali; i funzionari che le rilasciano i documenti in Argentina le attribuiscono la nazionalità jugoslava - essendo lei nata a Belgrado -; invece all'ambasciata argentina di Belgrado, la registrano come figlia di argentini. Poi, per una legge emanata durante la dittatura, perde la nazionalità argentina e figura per un periodo apolide; in seguito, diventa italiana, grazie ai nonni istriani che non avevano mai cambiato i loro passaporti ed erano rimasti italiani; infine, può riappropriarsi della sua nazionalità argentina:

Io ero argentina. A casa avevo sempre parlato in spagnolo. Le mie abitudini erano argentine. Mi piaceva l'asado, la grigliata argentina, il dulce de leche, quella marmellata di latte che a Belgrado ci mancava tanto, le empanadas, una specie di fagottini pieni di carne, formaggio, verdure, il mate, il flan, che suona così male tradotto con la parola budino. Non è che il cibo definisca l'identità di una persona, ma... Mi piacevano anche gli spaghetti, la mozzarella con pomodoro e basilico, pure i crauti e i peperoni grigliati, però si trattava di gusti che avevo acquisito, fatti miei, attribuito significati dopo, li avevo elaborati con coscienza. Gli altri, gli argentini, erano lì, erano quello che ero io e accompagnavano i miei gesti quotidiani. Come la lingua, lo spagnolo. ${ }^{19}$

16 «Negli anni continuai ad andare a Belgrado. Tutte le occasioni erano buone per vedere i miei amici. [...] io cercavo di spiegarle [all'amica Monica] le sensazioni che sentivo quando calpestavo quel territorio. L'appartenenza. Fare parte di un gruppo. Condividere. Mi sentivo viva» (ivi, p. 81).

17 Ivi, p. 114.

18 Ivi, pp. 91-93.

19 Ivi, p. 91. 
Dove, per inciso, sarà da notare un'ulteriore sottolineatura dell'importanza della lingua, oltre ai riferimenti al cibo, che ritornano spesso, nel libro, e, analogamente alle scelte linguistiche, sono funzionali a rendere il senso di un'identificazione e di un'appartenenza. Poco più avanti, però, di fronte all'Argentina trasformata dalla dittatura, questo senso di appartenenza sembra sfumare; anche gli anni di Trieste influiscono sul modo di pensare dell'io narrante, «in molte cose, anche se non lo volevo, ero italiana», fino a farla concludere: «Sono tornata, molte volte, ma alla fine non sono mai tornata. Non appartengo all'Argentina». ${ }^{20}$

Per mettere un po' d'ordine nel susseguirsi di appartenenze, di identificazioni, di sensazioni, che la scrittrice dichiara di volta in volta, può essere funzionale il ripensare alcune scelte dei genitori: nonostante la durezza delle loro vite, non hanno mai fatto avvertire alle figlie il peso dell'esilio, anzi, neppure loro l'hanno vissuto come tale; hanno sempre pensato, lo si è accennato, che bisognasse vivere secondo le abitudini dei paesi in cui si abitava; hanno cercato un altro mondo per essere se stessi, per non identificarsi con quello che lasciavano, ${ }^{21}$ per evitare di vivere, loro e le figlie, nel ricordo della dittatura militare:

Mio padre sentenziava: bisogna condividere il destino del paese in cui si vive! E io sempre immersa nella nostalgia. Non capivo. Che cosa voleva dire? L'ho capito dopo. Non voleva che le figlie vivessero nel ricordo tormentato di una dittatura militare. Meglio il presente del paese nuovo. Che curioso, mi ha sempre dato fastidio quella frase. Invece, era così semplice. $^{22}$

Colpisce, a questo proposito, la pregnanza, ma anche, al tempo stesso, l'essenzialità con cui la scrittrice allude ai traumi della sua vita - l'esilio, la migrazione - e a quanto hanno significato per lei nel tempo: sembra che abbia alla fine accolto l'invito del padre a non vivere perennemente nella nostalgia, a fare come i personaggi del suo Solo gli alberi hanno radici, che «continuano a vivere in mondi o in Stati che non ci sono più - come quando si dichiarano 'austriaci' perché nati nel defunto impero asburgico, mettendo in imbarazzo poliziotti, uffici e passaporti di tanti paesi - e che continuano ad amare pur senza nostalgia e amando anche gli altri luoghi del loro andare». ${ }^{23}$

\footnotetext{
20 Ivi, p. 91 e p. 96.

21 Ivi, p. 72.

22 Ivi, pp. 117-118.

23 C. Magris, Prefazione. Un mitteleuropeo sudamericano, cit., posizione 21.
} 
Ecco allora pochi accenni alla dittatura in Argentina, l'inquietante Ford Falcon senza targa sotto la casa dei Prenz, che segnala loro il pericolo che minaccia il padre, il silenzio sulle violenze e sulle uccisioni anche da parte degli amici, i funerali di un guerrigliero; non numerosi sono pure i riferimenti alle vicende della Jugoslavia, al percepibile malessere dei tardi anni Settanta, al suo progressivo 'svanire'. Gli ultimi capitoli del libro, Tomás e Ex, costituiscono invece una sorta di affondo sui due traumi: il primo racconta di un ragazzo argentino i cui genitori avevano scelto la lotta armata ed erano morti molto giovani; a lui ci era voluto del tempo per accettare la loro scelta e l'abbandono dei figli: solo più di trent'anni dopo, davanti alle ossa della madre, scomparsa venticinquenne, incinta e con due figli piccoli, ritrovate in una fossa comune, la incontra davvero, sente la connessione con lei ed è come se riuscisse finalmente a sentirsi, insieme alla nonna e alla sorella, 'intero' ${ }^{24}$

Il capitolo successivo, $E x$, registra una serie di stati d'animo della narratrice di fronte a quanto vede succedere in Jugoslavia, con le notizie drammatiche su croati e serbi, e la progressiva incomprensione delle reazioni di quelli che erano stati i suoi amici:

Io volevo continuare a essere jugoslava. Per un periodo andai a Belgrado. Nessuno poteva mettere in questione la mia lingua. Viaggiavo con una giornalista, traducevo per lei. Le cose fluivano. [...]. Assistevo all'esaltazione della mia gente stupita e distanziata. Il loro nazionalismo. Non avevo mai smesso di tornare a Belgrado. I miei amici più cari mi portavano in piazza, alle manifestazioni. [...] Non mi piaceva, non mi identificavo più. ${ }^{25}$

Assistere, poi, a uno scambio di prigionieri, serbi e croati, le dà l'impressione di rivedere scene del passato, vestiti come sono, i primi, con uniformi carcerarie uguali a quelle viste nei film sulla seconda Guerra Mondiale, nei campi di concentramento nazisti («nessun essere umano - commenta - deve soffrire tale umiliazione»). ${ }^{26}$ L'episodio, che viene filmato per essere dato in pasto alla «divulgazione mediatica», le consente anche di inserire una riflessione su una pretesa oggettività dell'informazione, che sottolinea essere invece tutt'altro che tale. Per la guerra di Bosnia, infine, «non ci son parole».

Significativamente, però, il racconto di Ana Cecilia Prenz si chiude con una suggestiva immagine, ancora in uno spazio di convivenza, multilingue e

24 A. C. Prenz Kopušar, Attraversando il fiume in bicicletta, cit., pp. 105-106.

25 Ivi, pp. 108-109.

26 Ivi, pp. 109-112. 
pluriculturale ${ }^{27}$ in cui il suo stesso riconoscersi latinoamericana le consente infine di assumere in sé e pacificare le sue diverse appartenenze:

Le cose cambiano, ma in fin dei conti neanche tanto. Sono in montagna. La signora zingara, con le gonne corte, non più lunghe e coprenti come un tempo, rallegra la mia mattinata. Ride. Lui invece fuma. Non fa altro accovacciato davanti al suo portale. Kako si, Ceco? Come stai, Ceca? È un serbo della Bosnia e mi chiama Ceca. Mai in un altro modo. La vicina a fianco mi dice Gospa, Signora, rispettosa, come sono gli sloveni, e mi porta ogni tanto del latte fresco. Un po' più in là c'è la mamma della ragazza musulmana, anche lei d'estate viene dalla Bosnia e lavora l'orto. Mi piace chiacchierare con lei. Ha la dolcezza dell'accettazione. Rosi, invece, mi parla dei fiori dell'Istria e del mare. Anche Monica mi fa visita. Parliamo di Trieste.

Mi ostino, o forse no. Sono fra le montagne e da questo luogo, forse ancora come un tempo, vivo il mio essere latinoamericana. ${ }^{28}$

27 Cfr. A. C. Crolla, Migración, autoficción y autotraducción en cruzando el río en bicicleta de Ana Cecilia Prenz Kopušar, cit., p. 204.

28 Ivi, p. 118. 